\title{
COMPROBACIÓN DEL EFECTO CICATRIZANTE DE Peperomia scutellaefolia $R$ et $P$. ASPECTOS BOTÁNICOS, QUÍMICOS Y FARMACOLÓGICOS
}

\author{
RUTH F. GUILleRMO', PABLO E. BONILLA R.' Y JORGE L. ARROYO A. ${ }^{2}$
}

\begin{abstract}
RESUMEN
En la presente investigación, se realizó una descripción de los caracteres morfológicos externos de la especie y la descripción de ciertos caracteres microscópicos en los diversos órganos de la planta. Se evaluó el efecto cicatrizante de Peperomia scutellacfolia $R$. et $P$. en forma de geles, mediante el método tensiométrico. Se utilizaron ratones albinos cepa Balb C 53 de 25 g de peso; y como tratamientos geles de Carbopol 940 al 5\%, 10\%, 20\%, y 30\% P/P de extracto vegetal, comparando los resultados con el grupo control y con el grupo tratado con un medicamento comercial. Se obtuvo mayor efecto cicatrizante con el gel al 5\%. Se aislaron e identificaron compuestos fenólicos y flavonoides por espectrofotometria ultravioleta-visible, espectrofotometría infrarroja y por reacciones químicas.
\end{abstract}

Palabras clave: Peperomia scutellaefolia R. et P., cicatrizante, método tensiométrico, flavonoides, espectrofotometría ultravioleta e infrarroja.

\begin{abstract}
SUMMARY
A description of the morphological external characteristics of Peperomia scutellaefolia R. et P. and some microscope details and the wound healing effect of vegetal specie Peperomia scutellaefolia $R$. et $P$. were evaluated and described in this research using tensiometric methods and the results were corroborated with histological studies in order to observe and analyze the histological evolution in each case. Batch Balb C 53 albine mice with $25 \mathrm{~g}$ of average weight were used and treated with Carbopol 940 gels with 5\%,10\%,20\% and $30 \% \mathrm{~W} / \mathrm{W}$ concentrations of this vegetal extract. The results obtained were compared with two groups: the control group (without treatment) and another group treated with a commercial medicament. A better wound healing effect with Peperomia's $5 \% \mathrm{~W} / \mathrm{W}$ concentration gel was obtained. Flavonoids and fenolic compounds were found and identified by ultraviolet and infrared spectrophotometry and coloration reactions.
\end{abstract}

Key words: Peperomia scutellaefolia R. et P., wound healing, tensiometric method, flavonoids, spectrophotometry ultraviolet and infrared.

\section{INTRODUCCIÓN}

En muchos sistemas de salud de América, Asia y Europa, es frecuente el uso de drogas vegetales $y$ fitomedicinas, como parte integral de la medicina convencional. En estos casos, basándose en la información médica tradicional, ha sido posible para la medicina científica validar la acción terapéutica y establecer los usos correctos de los recursos vegetales.

Las plantas del género Peperomia, son de uso frecuente como medicamentos en la sierra peruana, en casi toda la costa del Perú, y en algunas zonas

I Instituco de Investigación en Ciencias Farmacéuticas y Recursos Naturales «juan de Dios Guevara» Facultad de Farmacia y Bioquímica-Universidad Nacional Mayor de San Marcos.

2 Laboratorio de Farmacología Facultad de Medicina- Universidad Nacional Mayor de San Marcos. de la selva; en este sentido, es necesario estudiar sus efectos, con el fin de permitir su uso racional.

La especie Peperomia scutellaefolia R. et P., cuyo nombre vernacular es Munshu - Munshu (en quechua presenta dos significados: (i) ombligoombligo y (ii) bien redondo), esto en alusión a la forma de su tallo subterráneo generalmente esférico, que asemeja un ombligo. La especie crece en la sierra norte del Perú, en suelos secos y rocosos. Es utilizada tradicionalmente para curar males cardíacos, úlceras estomacales y heridas externas de la piel.

Se postula que la especie Peperomia scutellaefolia $R$. et P., posee influencia sobre la reparación tisular como cicatrizante externo por su gran contenido de compuestos fenólicos especialmente flavonoides. 
Los objetivos de la presente investigación fueron comprobar la acción cicatrizante externa atribuida a la especie Peperomia scutellaefolia R. et P., mediante ensayo farmacológico experimental, identificar las características botánicas de la planta que facilitarían la diferenciación de otras especies parecidas - en caso sea comercializada - entera o fragmentada, y comprobar los tipos de compuestos fenólicos que la especie Peperomia scutellaefolia R. et P. posee.

La cicatrización de las heridas cutáneas, se presenta como una masa de colágeno que se forma cuando no es posible reparar la necrosis de células parenquimatosas por regeneración; si se destruyen las células hasta la capa basal, de la dermis o epidermis, sucede una reparación con formación de cicatriz ${ }^{1}$. Todos los experimentos en cicatrización, buscan agentes que aceleren dicho proceso, el cual puede durar desde 3 meses hasta varios años para alcanzar un valor de resistencia a la tensión que se mantiene durante toda la vida y que suele ser del 70 a $80 \%$, respecto a la piel intacta ${ }^{2}$.

A nivel mundial, se ha evaluado el éxito de la cicatrización experimental por diversos métodos, por ejemplo, Raimondi y col. $^{3}$ evaluaron la actividad de los flavonoles contenidos en la especie Sedum telephium L.

\section{MATERIAL Y MÉTODOS}

\section{Estudio Botánico}

- Clasificación sistemática.

- Descripción macroscópica y microscópica de la especie.

- Corte transversal de la hoja.

- Corte transversal del pecíolo.

- Corte transversal del tallo subterráneo.

Los tallos subterráneos; crecen de manera silvestre en los distritos de Namora, Encañada, y Llacanora, en la provincia de Cajamarca, a una altitud entre 3000 y 4000 m.s.n.m.

La recolección, se realizó en el mes de septiembre cuando la especie se encontraba en etapa de floración. Peperomia scutellaefolia R. et P. crece en tierra seca y rocosa; el tallo subterráneo se encuentra entre 1 - 2 cm bajo tierra. Se limpió, secó, molió y se aImacenó en frascos oscuros.
Se empleó:

- Especie Peperomia scutellaefolia R. et P., planta completa y fresca.

- Trinocular stereo zoom. Marca: Beltec scientific. Model XLT-500B.

- Advanced grade microscopes. Marca: Beltec scientific. Model L2000A.

- Sistema de video-microscopía con conexión a PC. Beltec scientific. Modelo 480B.

- Colorante verde de malaquita ( $1 \%$ solución alcohólica), Colorante safranina ( $1 \%$ solución alcohólica), Sol. de $\mathrm{NaClO}$ (20\%), Gelatina glicerinada, Médula de sauco.

\section{Estudio Químico}

Se empleó:

- Espectrómetro ultravioleta visible. (UV - VIS) Perkin Elmer. Modelo: LAMBDA 40P

- Espectrómetro infrarrojo con Transformada de Foürier. (FT - IR) Nicolet. Modelo: IMPACT 410.

- Lámparas UV $254 \mathrm{~nm}$ y 366 nm.

- Balanza analítica Mettler.

- Material básico de vidrio.

El polvo seco fue macerado en solución hidroalcohólica (EtOH 96 $)$ por 7 días en oscuridad, luego fue filtrado con una gasa, el líquido filtrado fue concentrado, resultando el extracto hidroalcohólico total; a partir de este extracto se separaron cuatro fracciones por cromatografía en columna rápida (flash cromatographic). Se pesaron $5 \mathrm{~g}$ del extracto hidroalcohólico bruto, se agregaron $5 \mathrm{~g}$ de silicagel para cromatografía en columna, $0.05-0.2 \mathrm{~mm}$ y se mezcló hasta homogeneizar. Esta mezcla fue depositada en un embudo Buchner, sobre un papel de filtro, encima se colocó otro papel, el equipo fue armado según esquema, y con ayuda de la bomba de vacío, los solventes fueron extrayendo los metabolitos, en orden de polaridad creciente: $n$-hexano, cloroformo, metanol y finalmente agua destilada, se utilizaron $100 \mathrm{~mL}$ de cada solvente. Estos extractos fueron concentrados obteniéndose las fracciones nhexánica, clorofórmica, metanólica y acuosa.

- Ensayo de solubilidad del extracto total con solventes de polaridad creciente. 
- Marcha fitoquímica. Se realizaron las siguientes reacciones químicas en el extracto hidroalcohólico total: Reacción con $\mathrm{FeCl} 3$ (1\% en $\mathrm{H} 2 \mathrm{O})$; reacción con gelatina / $\mathrm{NaCl}$ (1g de gelatina $+100 \mathrm{ml} \mathrm{H} 2 \mathrm{O}+10 \mathrm{~g} \mathrm{NaCl}$ ); reacción de Shinoda; reacción de Dragendorff (Yoduro de Bismuto y Potasio); reacción de Mayer (Yoduro de Mercurio y Potasio); indice afrosimétrico o prueba de la espuma; reacción de LiebermannBurchard.

- Análisis cromatográfico. Los extractos obtenidos a partir de la cromatografía en columna rápida, se controlaron por cromatografía en capa fina analítica, usándose como soporte cromatoplacas de sílica gel $F 60$ de $20 \mathrm{~cm} \times 20$ $\mathrm{cm}$, de $2 \mathrm{~mm}$ de espesor y fases móviles adecuadas. Fueron revelados con los reactivos de Dragendorff, $\mathrm{FeCl}_{3}$, Liebermann-Burchard, $\mathrm{H}_{2} \mathrm{SO}_{4}$ concentrado, luz UV de $254 \mathrm{~nm}$ y $366 \mathrm{~nm}$. Se realizaron cromatografías sucesivas a escala preparativa, y se consiguió separar las fracciones en componentes individúales para su posterior evaluación espectrofotométrica.

- Análisis espectrofotométrico. Después de ser aisladas fueron analizadas las fracciones provenientes de los extractos metanólico y $n$-hexánico por espectroscopía ultravioleta y espectroscopía infrarroja. El espectro UV de las fracciones fue leído en $\mathrm{MeOH}$, y el espectro infrarrojo en láminas de $\mathrm{NaCl}$.

\section{Estudio Farmacológico}

Se empleó:

- Extracto hidroalcohólico total de Peperomia scutellaefolia R. et P.

- 96 ratones albinos machos, cepa Balb C 53, de 2 meses de edad, de alrededor de $25 \mathrm{~g}$ de peso.

- Dinamómetro (equipo de tensión con arena).

- Neomicina, bacitracina, glicina, L - cisteína, DL - treonina, (Cicatrín).

- Gel Carbopol 940, trietanolamina, propilenglicol, y $\mathrm{H} 2 \mathrm{O}$ desionizada.

- Crema depilatoria Opilca.

- Pentobarbital sódico (Halatal, medicamento veterinario)

- Estiletes $\mathrm{N}^{\circ} 11$, jeringas y agujas para inyección intraperitoneal.

Se trabajó con el extracto hidroalcohólico total.
Preparación de los geles:

(i) Se preparó una suspensión coloidal de Carbopol 940 al $1 \%$ en agua desionizada, con 24 horas de anticipación para lograr una buena hidratación, a un $\mathrm{pH}=3,0$.

(ii) Para la incorporación del principio activo se efectuó la disolución de este en una fracción de la solución coloidal, se agregaron unas gotas de propilenglicol para solubilizar el extracto y proteger de la deshidratación al gel.

(iii) Finalmente, se neutralizó con gotas de trietanolamina (99\%), homogeneizando hasta adquirir características de gel a $\mathrm{pH}=$ $6.5^{4}$

(iv) Forma de Dosificación. Con el extracto hidroalcohólico total seco, se prepararon geles al $5 \%, 10 \%, 20 \%, 30 \%, \mathrm{P} / \mathrm{P}$ en una base bioadhesiva de ácido poliacrílico (Carbopol 940 ) al $1 \%$ y gel con solo la base.

\section{Test de cicatrización}

Se fundamenta en la medición de la fuerza de tensión (medida en gramos), necesaria para abrir una herida de $1 \mathrm{~cm}$ de longitud producida en el lomo de ratón. Modelo de referencia de Howes et al. ${ }^{5}$

Técnica operatoria. Se utilizó el método de Vaisberg y col. ${ }^{6,7}$ con 96 ratones albinos machos, cepa Balb C 53, de 2 meses de edad, $25 \mathrm{~g}$ de peso provenientes del bioterio del Centro Nacional de Producción de Biológicos, los cuales fueron distribuidos al azar en 8 grupos de 12 cada uno. Se mantuvieron en observación por 48 horas, verificándose la condición óptima de los ratones para el estudio, luego se les depiló la mitad inferior del lomo, después de 24 horas, al no observarse irritaciones en la piel, se realizaron incisiones de $1 \mathrm{~cm}$ de longitud en el tercio inferior del lomo, paralelo a la columna lumbar.

Posteriormente, se administraron los tratamientos cada 12 horas por 72 horas, reservando al grupo control que no recibió tratamiento. Se mantuvo la misma alimentación, ventilación, y $\mathrm{T}^{\circ}$ en todos los grupos. Después de las 72 horas se sacrificaron los animales con una sobredosis de pentobarbital sódico por vía intraperitoneal; luego se realizó la medición de los gramos (g), necesarios para abrir cada herida cicatrizada con un dinamómetro (utilizándose arena en el dinamómetro, para generar la fuerza de tensión sobre la herida). 


\section{RESULTADOS}

\section{Estudio Botánico}

Clasificación sistemática. Realizada en el Museo de Historia Natural «Javier Prado», según el sistema de clasificación de Engler \& Prantl, modificado por Melchior en 1964, como sigue:

\section{DIVISIOON: ANGIOSPERMAE}

CLASE:

\section{DICOTILEDONEAS}

SUBCLASE: ARQUICLAMIDEAS

ORDEN:

$$
\text { PIPERALES }
$$

FAMILIA:

\section{PIPERACEAE}

GENERO:

PEPEROMIA

ESPECIE:

Peperomia scutellaefolia $\mathrm{R}$. et $\mathrm{P}$.

Nombre Vulgar: Munshu-Munshu.

Descripción macroscópica y microscópica de Peperomia scutellaefolia $\mathrm{R}$. et $\mathrm{P}$.

Es una planta herbácea, el tallo es subterráneo, de forma generalmente esferoide, achatado en la parte superior e inferior. Aunque también puede ser algo cilíndrico o alargado en algunos ejemplares, especialmente en las plantas más viejas. En general, es bastante semejante a una papa pequeña pero sin yemas laterales, puede medir hasta $4 \mathrm{~cm}$ de diámetro y $3 \mathrm{~cm}$ de altura, posee pequeñas raíces que suelen desarrollarse a partir de la mitad inferior del ta1lo. En la parte superior presenta una yema, como hendidura, a partir de la cual se desarrolla el escapo.

Con hojas peltadas de color verde brillante el haz $y$ color rojizo el envés, totalmente glabras, de forma elíptica a redonda, de hasta $2 \mathrm{~cm}$ de diámetro, margen liso, con una ligera emarginación al final de la nervadura principal, la nervadura principal parte del punto de inserción del pecíolo, que puede insertarse desde el centro de la lámina foliar, hasta los $2 /$ 3 de la hoja, las ramificaciones de la nervadura son tenues y se observan sólo en el envés.

Los pecíolos son largos y delgados, midiendo entre 3 hasta $7 \mathrm{~cm}$ de largo y aproximadamente $1.0 \mathrm{~mm}$ de diámetro, glabros, de color rojizo, excepto en la porción inferior donde se unen al tallo que es amarillo muy claro.

Las inflorescencias son espadiciformes, miden hasta $6 \mathrm{~cm}$ de largo, de flores diminutas, color verde claro, el pedúnculo es apenas diferente del pecíolo y mide entre $3-8 \mathrm{~cm}$, también es de color rojizo y cambia al amarillo justo antes de empezar la espádice. Cuando las flores son inmaduras se observan 2 brácteas opuestas de color rojizo oscuro, conforme van madurando las flores, las brácteas cambian su color a crema, y las flores adultas se ubican en la parte inferior de la espádice. En la inflorescencia se observan tres zonas: inferior, de flores maduras, que adquiere color amarillo, intermedia, de flores con brácteas redondas a ovaladas pegadas a la flor, de color rojizo oscuro, y superior, de flores inmaduras, que aún no desarrollan brácteas coloreadas, muy pequeñas y apiñadas unas a otras.

\section{Estudio Químico}

Resultado de la marcha de solubilidad del extracto hidroalcohólico total, bastante soluble en metanol y agua.

Resultados de la marcha fitoquímica del extracto hidroalcohólico total, se ha determinado presencia abundante de compuestos fenólicos, flavonoides, taninos y alcaloides.

Resultados del análisis cromatográfico, se realizaron cromatografías en capa fina de las fracciones resultantes de la cromatografía rápida. Cuadros 1 y 2.

\section{1.- Fracción metanólica}

TABLA 1. SEPARACIÓN DE LA FRACCIÓN METANÓLICA TOTAL POR CROMATOGRAFÍA EN CAPA FINA (Sistema de solventes: $\mathrm{CHCl}: \mathrm{MeOH}$ 15:1)

\begin{tabular}{|l|l|l|l|l|l|}
\hline Subfracción & Rf & Visible & Fluorescencia a la Luz UV & $\mathrm{FeCl}_{3}$ & Rvo. Dragendorff \\
\hline Metanólica I & 0.05 & marrón beige & púrpura amarillo & + & + \\
Metanólica II & 0.20 & beige & verde amarillento & + & + \\
Metanólica III & 0.27 & beige claro & verde intenso & + & + \\
Metanólica IV & 0.41 & berge claro & blanco azulado & - & + \\
Metanólica V & 0.73 & - & púrpura & + & + \\
Metanólica VI & 0.81 & - & celeste & - & + \\
Metanólica VII & 0.92 & crema beige & blanco verduzco & + & - \\
\hline
\end{tabular}


a) La fracción metanólica I del Cuadro 1 con $\mathrm{Rf}=$ 0.05 , se eluyó y se recromatografió con un sistema de solventes: EtOH : $\mathrm{H} 2 \mathrm{O}$ 3:1, obteniéndose a su vez cuatro subfracciones, $\mathrm{F}$ I-1, FI-2, FI-3 y FI-4. La subfracción metanólica $\mathrm{I}-1$ con $\mathrm{Rf}=0.20$, se recromatografió con un sistema de solventes EtOH : $\mathrm{H} 2 \mathrm{O} 3: 1$, obteniéndose tres subfracciones, con la subfracción metanólica I-1.1 se realizaron lecturas para obtener sus espectros UV-visible e IR.

b) La fracción metanólica III del Cuadro 1 con Rf $=0.27$ se eluyó y se recromatografió en sistema de solventes: $\mathrm{CHCl3}$ : $\mathrm{MeOH}$ 10:1 obteniéndose cinco subfracciones. Con la subfracción metanólica III-5 se realizaron lecturas para obtener sus espectros UV-visible e IR.

c) La fracción metanólica $\mathrm{V}$ del Cuadro 1 con Rf $=0.73$; se eluyó y se recromatografió en sistema de solventes: B.A.W. (n-butanol, ácido acético, agua 4:1:5) obteniéndose dos subfracciones: FM V-1 y FM V-2, se realizaron lecturas para obtener sus espectros UV-visible e IR.

2.- Fracción n-hexánica

TABLA 2. SEPARACIÓN DE LA FRACCIÓN N-HEXÁNICA POR CROMATOGRAFÍA EN CAPA FINA (Sistema de solventes: $\mathrm{CHCl} 3: \mathrm{MeOH}$ 20:1)

\begin{tabular}{|l|l|l|l|l|c|l|}
\hline Subfracción & Rf & Visible & Luz UV & $\mathrm{FeCl}_{3}$ & Liebermann Burchard & $\mathrm{H}_{2} \mathrm{SO}_{4}+\mathrm{LuzUV}$ \\
\hline Hexánica I & 0.79 & beige & fl. amarillo & + & & - \\
Hexán n i c a & 0.89 & beige claro & fl. verde celeste & + & & amarillo \\
II/III & 0.92 & beige & fl. púrpura & + & & - \\
Hexánica IV & & & & & & azul intenso \\
& & & & & & \\
\hline
\end{tabular}

$(+)=$ presencia,$(-)=$ ausencia

Seguidamente se realizaron lecturas para obtener sus espectros UV-visible e IR.

\section{Estudio Farmacológico}

Test de cicatrización

TABLA 3. RESULTADOS DEL TEST DE CICATRIZACIÓN (MÉTODO TENSIOMÉTRICO)

\begin{tabular}{|l|c|c|c|c|c|c|}
\hline TRATAMIENTO & $\mathbf{N}$ & $\begin{array}{c}\text { Valor } \\
\text { medio (g) }\end{array}$ & $\begin{array}{c}\text { Desviación } \\
\text { estándar (g) }\end{array}$ & $\begin{array}{c}\text { Error } \\
\text { estándar (\%) }\end{array}$ & $\begin{array}{c}\text { Eficacia } \\
\text { decicatrización } \\
(\%)\end{array}$ & $\begin{array}{c}\text { Significancia } \\
(\mathbf{p})\end{array}$ \\
\hline Lesión no & & & & & & \\
tratada & 10 & 15.9 & 1.7288 & 0.5467 & 7.10 & $\mathrm{P}>0.05$ \\
Gel & 10 & 20.5 & 2.0683 & 0.6540 & 9.10 & $\mathrm{P}>0.05$ \\
Gel 5\% & 10 & 54.6 & 1.7764 & 0.5617 & $24.25^{*}$ & $\mathrm{P}<0.0001$ \\
Gel 10\% & 10 & 40.6 & 4.5509 & 1.4391 & $18.03^{*}$ & $\mathrm{P}<0.0001$ \\
Gel 20\% & 10 & 44.3 & 4.7621 & 1.5059 & $19.70^{*}$ & $\mathrm{P}<0.0001$ \\
Gel 30\% & 10 & 47.6 & 4.4522 & 1.4079 & $21.14^{*}$ & $\mathrm{P}<0.0001$ \\
Cicatrín & 10 & 43.6 & 2.6331 & 0.8327 & $19.36^{*}$ & $\mathrm{P}<0.0001$ \\
Piel intacta & 10 & 225.2 & 12.2547 & 3.8753 & 100.00 & $\mathrm{P}<0.0001$ \\
\hline
\end{tabular}

(\%) Eficacia de = (gramos necesarios para abrir la cicatriz tratada) $\times 100$ cicatrización (gramos necesarios para abrir la piel intacta) 


\section{Discusión}

La descripción macroscópica de Peperomia scutellaefolia $R$. et $P$. conocida con el nombre común de Munshu-Munshu, corresponde a la literatura disponible ${ }^{8,9}$, como características propias de la especie que permiten diferenciarlas de otras Peperomias como Peperomia peruviana, puede mencionarse: el pecíolo pigmentado, las hojas peltadas que tienden a adoptar forma de conos, brillantes en el envés. En el cormo (tallo subterráneo) se observa que las raíces se desarrollan a partir de la mitad inferior del tallo. Al ser cortado fresco, el cormo despide un olor característico que hace recordar al jengibre, y las superficies son pegajosas al tacto.

Las características reportadas de la hoja se observaron en 2 cortes histológicos: del pecíolo y de la lámina foliar. En la lámina foliar se observan características propias de la especie, como la epidermis pluriestratificada. Se observan también idioblastos (con contenido amarillo e hidrosoluble), además de algunos cristales de oxalato de calcio, la epidermis inferior presenta células que contienen pigmentos violetas. La lámina foliar es glabra en el haz y el envés. El corte histológico del pecíolo fue tomado de la parte media, donde se observaron células pigmentadas de violeta en la parte externa.

En caso de que la especie fuera comercializada fragmentada, se podría identificar por la peridermis, los abundantes cristales y las células con contenido amarillo hidrosoluble (probablemente flavonoides), características observables en el tallo subterráneo.

Con respecto al estudio fitoquímico de los extractos: la marcha de solubilidad indica que se obtendría una mayor cantidad de extracto vegetal utilizando una mezcla de solventes polares como $\mathrm{MeOH}+\mathrm{H}_{2} \mathrm{O}$.

La marcha fitoquímica permite establecer que Peperomia scutellaefolia $R$. et $P$. contiene principalmente compuestos fenólicos (flavonoides, taninos, etc.) y alcaloides. Esto concuerda con los datos reportados para otras especies del género Peperomia también presentes en el Perú ${ }^{10}$.

Los cromatogramas en capa fina de los extractos mostraron que las fracciones metanólica y hexánica, poseen interés por contener gran cantidad de compuestos fenólicos y flavonoides.

\section{Elucidación estructural propuesta}

Se proponen estructuras químicas al realizar el análisis del comportamiento químico y fisicoquímico11,12 a través de los espectros Ultravioleta-viśible e Infrarrojo de las fracciones aisladas mediante técnicas cromatográficas.

1. Fracción metanólica I-1.1; lecturas obtenidas: UV-visible: $\lambda^{\mathrm{MeOH}} 236(\mathrm{~h}), 282,314(\mathrm{~h}) \mathrm{nm}$; IR: $v \mathrm{~cm}^{-1} 3409(-\mathrm{OH}), 1645(>\mathrm{C}=\mathrm{O}), 1074(\mathrm{C}-\mathrm{H}$ aromático), $2850-3000\left(-\mathrm{CH}_{2} \mathrm{y}-\mathrm{CH}_{3}\right.$ alifáticos $)$.

Comparando los espectros UV- visible con los reportados por Mabry ${ }^{10}$, correspondería al dihidroflavonol glucosilado (+) Fustin- 3-Oglucopiranósido.

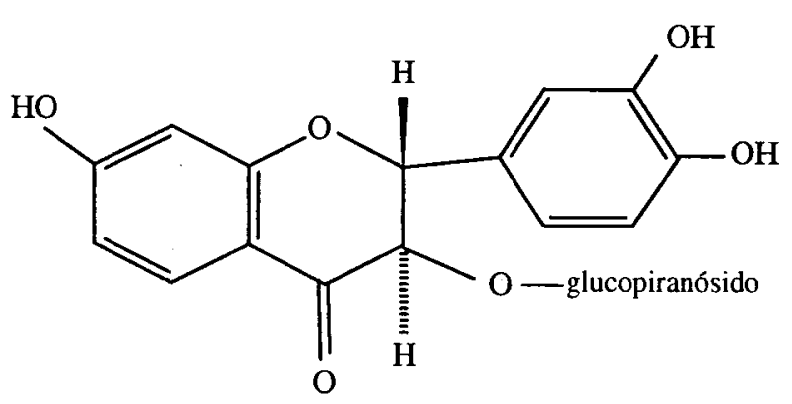

Fig. 1. (+) Fustin-3-O-glucopiranósido

2. Fracción metanólica III-5; lecturas obtenidas: UV-visible: $\lambda^{\mathrm{MeOH}} 226,282(\mathrm{~h}) \mathrm{nm}$; IR: $v \mathrm{~cm}^{-1}$ $3427(-\mathrm{O}-\mathrm{H}), 2952,2926,2840\left(-\mathrm{CH}_{2}\right.$ y $-\mathrm{CH}_{3}$ alifáticos), 1644, 1729 (>C=O de ácido carboxílico), 1456 (-C=C- aromático), 9701220(-C-H aromático)

Con los datos anteriores de los espectros UVvisible e IR se deduce que correspondería a un compuesto fenólico monocíclico simple de tipo fenilpropanoide. (ácido 2-en-2-metil-3-p hidroxi fenilpropanoico)

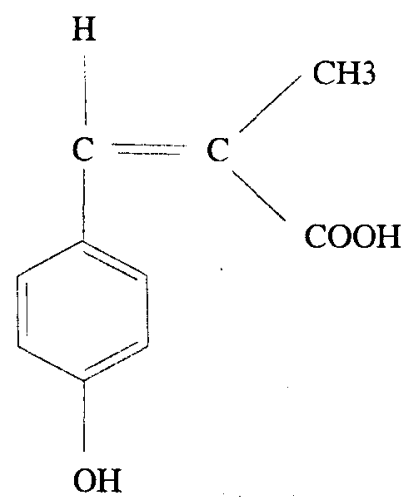

Fig. 2. Ácido 2-en-2-metil-3-phidroxifenilpropanoico 
3. Fracción metanólica V-1; lecturas obtenidas: UV-visible: $\lambda^{\mathrm{MeOH}} 205(\mathrm{~h}), 258 \mathrm{~nm}$; IR: $v \mathrm{~cm}^{-1}$ $3416(-\mathrm{OH}) ; 1638(>\mathrm{C}=\mathrm{O})$, luego de analizar correspondería a un fenilpropanoide (ácido 3fenilpropanoico).<smiles>O=C(O)CCc1ccccc1</smiles>

Fig. 3. Àcido 3-fenilpropanoico

4. Fracción metanólica V-2; lecturas obtenidas: UVvisible: $\lambda^{\text {MeOH }} 254,297(\mathrm{~h}), 330(\mathrm{~h}) \mathrm{nm}$; IR: $\mathrm{v} \mathrm{cm}$ $3447(-\mathrm{OH}) ; 1638(>\mathrm{C}=\mathrm{O})$, por los espectros UVvisible se trataría de una isoflavona con estructura similar al pratensein, sería la isoflavona 7,3'-dihidroxi-5,4'-dimetoxiisoflavona.<smiles>CCOc1ccc(-c2coc3cc(O)cc(OC)c3c2=O)cc1O</smiles>

Fig. 4. $7,3^{\prime}$-dihidroxi-5, $4^{\prime}$-dimetoxiisoflavona

5. Fracción n-hexánica-I; lecturas obtenida: UVvisible: $\lambda^{\mathrm{MeOH}} 256,283(\mathrm{~h}), 330(\mathrm{~h}) \mathrm{nm}$; IR: v $\mathrm{cm}^{-1} \quad 2923(\mathrm{C}-\mathrm{H}) ; 1748(>\mathrm{C}=\mathrm{O})$; 1463 (deform. $\mathrm{C}=\mathrm{C}$, aromático); 1372(estiramiento $\mathrm{C}-\mathrm{O}$, de fenoles); 717, 705(C-H aromático)

Resultando el compuesto 7,4'-dihidroxi-5,3'dimetoxiisoflavona.

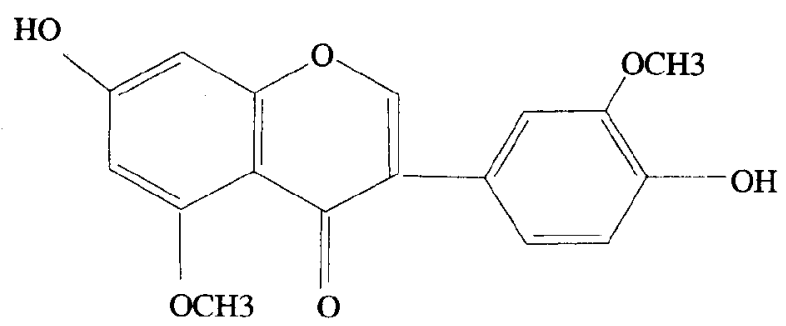

Fig. 5. 7,4'-dihidroxi-5, $3^{\prime}$-dimetoxiisoflavona
6. Fracción n-hexánica II; lecturas obtenidas: UVvisible: $\lambda^{\mathrm{MeOH}} 259,327 \mathrm{~nm}$; IR: $v \mathrm{~cm}^{-1} 3420(-$ $\mathrm{OH}) ; 1638(>\mathrm{C}=\mathrm{O})$. La fracción n-hexánica-II correspondería a la isoflavona Texasin -7-Oglucopiranósido (6-hidroxi-7-Oglucopiranosil-4'-metoxiisoflavona)<smiles>CCCCOc1cc2occ(-c3ccc(OC)cc3)c(=O)c2cc1O</smiles>

Fig. 6. Texasin -7-O-glucopiranósido

7. Fracción n-hexánica III; lecturas obtenidas: UV-visible: $1^{\mathrm{MeOH}} 264,318(\mathrm{~h}), 335(\mathrm{~h}) \mathrm{nm}$; IR: $\mathrm{n}$ $\mathrm{cm}^{-1}$ 2909(-C-H de aldehído -CHO); 1722 $(>\mathrm{C}=\mathrm{O}$, grupo carbonilo); $1457(\mathrm{C}=\mathrm{C}$, aromático). Analizando los espectros UV e IR de la fracción n-hexánica III; este compuesto correspondería a un fenilpropanoide (2-en-3fenilpropionaldehido)<smiles>O=C/C=C/c1ccccc1</smiles>

Fig. 7. 2-en-3-fenilpropionaldehido

8. Fracción n-hexánica IV; lecturas obtenidas: UVvisible: $\lambda$ MeOH $267,326 \mathrm{~nm}$; IR: $v \mathrm{~cm}^{-1} 3429(-$ $\mathrm{OH}) ; 2962-2845(\mathrm{C}-\mathrm{H}) ; 1645(>\mathrm{C}=\mathrm{O}) ; 1457$ 1372( $\mathrm{C}=\mathrm{C}$, aromático)

Correspondería al 5-hidroxi-7-O-glucopiranósido-6,4'-dimetoxiisoflavona.<smiles>COc1ccc(-c2coc3cc(OC)c(OC)c(O)c3c2=O)cc1</smiles>

Fig. 8. 5-hidroxi-7-O-glucopiranósido-6,4'dimetoxiisoflavona 
De la evaluación espectrofotométrica de las fracciones aisladas, se encontró que estas contenían flavonoides derivados del núcleo de la isoflavona y un derivado del núcleo del dihidroflavonol, estos compuestos se generan por la ruta biogenética del shikimato y del acetato-malonato. Además de los flavonoides hallados como tales, se encontraron sus precursores: los fenilpropanoides que provienen de la ruta del shikimato.

De los ensayos para evaluar la actividad cicatrizante se observó que todos los geles formulados a base del extracto de Peperomia scutellaefolia R. et P; presentan actividad terapéutica como cicatrizantes externos, esto se refleja en una mayor velocidad de reparación en los primeros días de curación de la herida, posteriormente las heridas continúan con sus etapas normales de acumulación y remodelación del colágeno, para finalmente alcanzar una resistencia de aproximadamente el $80 \%$ de la piel intacta. En orden de mayor a menor actividad se encuentran: gel $5 \%$, gel $30 \%$, gel $20 \%$, y por último el gel $10 \%$. Este efecto podría deberse a la presencia de flavonoides contenidos en las plantas de la familia Piperaceae, como reporta Villegas et al. ${ }^{7}$, que investigaron la actividad cicatrizante de Peperomia galioides H.B.K y Arroyo et al. ${ }^{13}$, que investigaron la actividad cicatrizante de Piper angustifolium.

\section{CONCLUSIÓN}

La especie Peperomia scutellaefolia R. et P., presenta actividad terapéutica como cicatrizante externo en la forma farmacéutica de gel. El tratamiento con mayor eficacia fue el gel al 5\%. Tomando como valor referencial de $100 \%$ a la resistencia a la tensión de la piel intacta. Estadísticamente se observó que los niveles de resistencia a la tensión alcanzados con los tratamientos de Peperomia scutellaefolia R. et $P$., presentan todos una diferencia significativa al ser comparados con el tejido no tratado, con una significancia de: $\mathrm{p}<0.0001$.

\section{REFERENCIAS BIBLIOGRÁFICAS}

1. Parakrama Chandrasoma, Taylor CR. Patología General. $2^{\mathrm{a}}$ Edición. Ed. Manual Moderno; México D.F 1998.
2. Robbins, Cotran RS, Kumar. Inflamación, Patología estructural y funcional. $4^{a}$ Edición. Volumen I. Editorial Interamericana; México D.F 1990.

3. Raimondi L, Banchelli G, Dalmazzi D, Mulinacci N, Romani A, Vincierri F et al. Sedum telephium L. Polysaccharide Content Affects MRC5 Cell Adhesion to Laminin and Fibronectin. J. Pharm. Pharmacol. 2000; 52: $585-591$.

4. Fauli I, Trillo et al. Tratado de Farmacia Galénica. Luzan S. S.A. de Ediciones; Madrid 1993.

5. Howes E, Sooy J, Harvey S. The healing of wound as determined by their tensile strength. J.A.M.A. 1929; 42(5).

6. Vaisberg JA, Milla $\mathrm{M}$, Planas $\mathrm{M}$, Cordova JL, Agusti ER, Ferreira R, Mustiga MC. Taspina is the cicatrizant principle in sangre de grado extracted from Croton lechleri. Planta Médica. 1989, 55: 140-143.

7. Villegas LF, Fernández ID, Maldonado H, Torres R, Zavaleta A, Vaisberg AJ, et al. Evaluation of the wound. - healing activity of selected traditional medicinal plants from Perú. J of Ethnopharmacol 1997; 55(3): 193 - 200.

8. MacBride JF. Flora of Perú. Publication of Field Museum of Natural History. Botanical Series Vol. XIII, part II N ${ }^{\circ} 1$.Chicago; 1937.

9. www.peperomiapage.com

10. Lozano, N. y col. Estudio de las especies Peperomias de la familia Piperaceae. Scienta Omni. 1997; 1(3):71-81.

11. Mabry T, Markham $K$ and Thomas $M$. The Systematic Identification of Flavonoids. New York. Springer Verlag; 1970.

12. Lock O. Investigación Fitoquímica. Métodos en el Estudio de Productos Naturales. Fondo Editorial P.U.C.P. Lima; 1994.

13. Arroyo J, Pareja B, Raez J. Efecto cicatrizante de Piper angustifolium R. \& P. sobre lesiones de piel inducidas en animales de experimentación. Folia Dermatológica Peruana 1999; 10 (1). 